\title{
Autonomous evolution of electron speeds in a thermostatted system: exact results
}

\author{
F. Bonetto, \\ School of Mathematics, Georgia Institute of Technology, Atlanta GA 30332, USA \\ N. Chernov*, \\ Department of Mathematics, University of Alabama at Birmingham, Birmingham AL 35294, USA \\ A. Korepanov, \\ College of Engineering, Mathematics and Physical Sciences, University of Exeter, Exeter, UK \\ J.L. Lebowitz. \\ Departments of Mathematics and Physics, Rutgers University, Piscataway NJ 08854, USA
}

31 August 2018 (upd. 26 December 2018)

\begin{abstract}
We investigate a dynamical system consisting of $N$ particles moving on a $d$-dimensional torus under the action of an electric field $E$ with a Gaussian thermostat to keep the total energy constant. The particles are also subject to stochastic collisions which randomize direction but do not change the speed. We prove that in the van Hove scaling limit, $E \rightarrow 0$ and $t \rightarrow t /|E|^{2}$, the trajectory of the speeds $v_{i}$ is described by a stochastic differential equation corresponding to diffusion on a constant energy sphere. This verifies previously conjectured behavior.

Our results are based on splitting the system's evolution into a "slow" process and an independent "noise". We show that the noise, suitably rescaled, converges to a Brownian motion, enhanced in the sense of rough paths. Then we employ the Itô-Lyons continuity theorem to identify the limit of the slow process.
\end{abstract}

\section{Introduction}

Many-particle dynamical systems in which different quantities evolve on different time scales are common in nature. This situation arises when the microscopic degrees of freedom come to some local equilibrium (stationary) state characterized by parameters which vary slowly in time. This leads to autonomous macroscopic equations, such as the Navier-Stokes equation, with or without stochastic terms. Such equations are generally very hard to derive rigorously, especially in situations which involve deterministic dynamics. In this note we continue our investigation of such a system of $N$ interacting particles with both deterministic and stochastic dynamics which conserves the total kinetic energy.

*Deceased 
The system we consider is a variation of the Drude-Lorentz model of electrical conduction in a metal [1]. It consists of $N$ particles (electrons) in a $d$-dimensional torus under the action of a constant external field $E$. The particles undergo elastic collisions with fixed or random scatterers, which change directions of the velocities $\left\{p_{k}\right\}$ but not the speeds $\left\{\left|p_{k}\right|\right\}$, and are subject to a Gaussian thermostat which keeps the total kinetic energy of the system constant. The thermostat introduces dynamical interactions between the particles.

We have studied this system extensively, in $d=2$, via numerical simulations and approximate analytical methods, using various models for the elastic collisions [5, 4, 6, 7]. We have argued there that in all collision models, for a weak field $E$ the evolution splits into a fast and slow parts which evolve essentially independently, with the slow part satisfying an autonomous diffusive equation. We were however unable to prove this in a rigorous way: see Section 5 ,

Here we show, for the first time, in a rigorous mathematical way, using the simplified collision model, that the long time weak field evolution of the properly scaled system (van Hove scaling) is described by an autonomous SDE, driven by an $N$-dimensional Brownian motion. To do this we apply the theory of "rough paths", pioneered by Lyons (see e.g. [16]). We follow and adapt the approach of Kelly and Melbourne [13] for nonuniformly hyperbolic dynamical systems. We find a way to decompose the evolution of the velocities $\left\{p_{k}\right\}$ into "fast" and "slow" components, with the fast component uncoupled from the slow.

Remark 1.1. A straightforward decomposition would be $p_{k} \mapsto\left(v_{k}, \omega_{k}\right)$, where $v_{k}=\left|p_{k}\right|$ and $\omega_{k}=$ $p_{k} /\left|p_{k}\right|$. This corresponds to an intuition of quickly changing directions and slowly changing speeds. However, in such a decomposition, the evolution of $\left\{\omega_{k}\right\}$ depends on $\left\{v_{k}\right\}$ : between collisions, the particles change directions, and the lower a particle's speed, the faster its direction changes. The influence of $\left\{v_{k}\right\}$ on $\left\{\omega_{k}\right\}$ causes substantial problems: the topic of rough differential equations with noise coupled to the solution is an unexplored area. (Though some related progress has been made in dynamical systems [9, 10, 11].) We use a different decomposition, which may look technical and artificial but gives independent noise.

To make our approach work, we make the following simplifying assumptions:

- we assume that the collision rate of particle $k$ is independent from its speed $v_{k}$. In the kinetic theory of gases, these are referred to as "Maxwellian" collisions;

- at each collision, the outgoing direction $\omega$ of the colliding particle is selected uniformly on the unit sphere $S^{d-1}$ in $\mathbb{R}^{d}$.

The paper is organized as follows. In Section 2 we give a precise description of the model and state our results. Section 3 contains the main idea and strategy of the proof of our main result while Section 4 contains a technical adaptation of [13] to our situation, used in Section 3. Finally, in Section 5 we discuss the connection of this work with previous work in this ongoing research. In Appendix $\mathrm{A}$ we give a standard example where the solution of a differential equation is not a continuous function of a driving signal, and in Appendix B we show that an $n$-particle diffusion on the sphere of radius $\sqrt{n}$ corresponds, as $n \rightarrow \infty$, to an Ornstein-Uhlenbeck process for the motion of a single particle. 


\section{$2 \quad$ Model and Results}

We consider a system formed by $N$ particles moving on a torus $\mathbb{T}^{d}, d \geq 2 \sqrt[1]{1}$ with positions $q_{k}$ and velocities $p_{k}=\dot{q}_{k}$. The particles move under an electric field $E$ and a Gaussian thermostat, which preserves the total energy, so $U=\sum_{k}\left|p_{k}\right|^{2}$ is constant [5, 4, 6, 7]:

$$
\left\{\begin{array}{l}
d q_{k}=p_{k} d t \\
d p_{k}=\left(E-\frac{\sum_{j} E \cdot p_{j}}{U} p_{k}\right) d t
\end{array}\right.
$$

In addition each particle experiences random collisions, independent of the other particles. Collisions are driven by Poisson processes with rate $\lambda>0$, the same for all particles. At a collision, a particle's direction $p_{k} /\left|p_{k}\right|$, which is a point on the unit sphere $S^{d-1}$ in $\mathbb{R}^{d}$, changes randomly and uniformly on $S^{d-1}$, while the speed $\left|p_{k}\right|$ is preserved.

Suppose that $E=\varepsilon \hat{n}$, where $\hat{n} \in \mathbb{R}^{d}$ is a fixed unit vector, and $\varepsilon>0$. Let $v_{k}=\left|p_{k}\right|$. Let the initial conditions $p_{k}(0)$ and $q_{k}(0)$ be fixed. Then $v=\left(v_{1}, \ldots, v_{N}\right)$ is random process with continuous sample paths.

Remark 2.1. In our model, positions of particles affect neither velocities between collisions nor collisions themselves. Working with the velocities, the positions can be safely ignored.

Heuristic arguments [5] show that for small $\varepsilon$, the time changed process $v^{\varepsilon}, v^{\varepsilon}(t)=v\left(\varepsilon^{-2} t\right)$, behaves like a nontrivial stochastic process. In this paper, we describe this behavior rigorously.

Our main result is:

Theorem 2.2. As $\varepsilon \rightarrow 0$, the time changed processes $v^{\varepsilon}, v^{\varepsilon}(t)=v\left(\varepsilon^{-2} t\right)$, converge weakly to the solution $v^{0}$ of the Itô stochastic differential equation

$$
\begin{aligned}
d v_{k}^{0} & =\delta^{1 / 2}\left[d W_{k}-v_{k}^{0} \frac{\sum_{j} v_{j}^{0} d W_{j}}{U}\right]+\delta\left[\frac{d-1}{2 v_{k}^{0}}-\frac{(N d-1) v_{k}^{0}}{2 U}\right] d t, \\
v_{k}^{0}(0) & =\left|p_{k}(0)\right| .
\end{aligned}
$$

Here $\delta=2 \lambda^{-1} d^{-1}$ and $W_{1}, \ldots, W_{N}$ are standard independent Brownian motions. The weak convergence is in the $C^{0}\left([0, \infty), \mathbb{R}^{N}\right)$ topology.

Remark 2.3. Since the processes $v^{\varepsilon}$ have continuous sample paths in $\mathbb{R}^{N}$, it is natural to think of them as random elements of the space $C^{0}\left([0, \infty), \mathbb{R}^{N}\right)$ of continuous functions with the usual topology of uniform convergence on compact sets.

The weak convergence of $v^{\varepsilon}$ to $v^{0}$ in $C^{0}\left([0, \infty), \mathbb{R}^{N}\right)$ topology means that $\mathbb{E} f\left(v^{\varepsilon}\right) \rightarrow \mathbb{E} f\left(v^{0}\right)$ for every continuous test function $f: C^{0}\left([0, \infty), \mathbb{R}^{N}\right) \rightarrow \mathbb{R}$. Weak convergence is also known as convergence in distribution and convergence in law.

Remark 2.4. The evolution of speeds $\left\{v_{k}^{0}\right\}$ can be described by a much simpler SDE. There exists a $d N$-dimensional process $u^{0}=\left(u_{1}^{0}, \ldots, u_{N}^{0}\right)$, with each $u_{k}^{0}$ a $d$-dimensional process and $v_{k}^{0}=\left|u_{k}^{0}\right|$ at all times. The process $u^{0}$ is a diffusion on the sphere $\left|u^{0}\right|^{2}=U$ which can be written as a solution of a Stratonovich SDE

$$
d u^{0}=\left(I-\frac{u^{0}\left(u^{0}\right)^{*}}{U}\right) \circ d W
$$

Here $W$ is a $d N$-dimensional Brownian motion with covariance matrix $2 \lambda^{-1} d^{-1} I$. See Section 3 , specifically Theorem 3.3, for details.

\footnotetext{
${ }^{1}$ See Remark 2.5 for $d=1$.
} 
Remark 2.5. Theorem 2.2 is restricted to $d \geq 2$ : with $d=1$, SDE (2) gives a wrong process which allows $v_{k}$ to become negative. Remark 2.4, however, fully applies to all $d \geq 1$. In our proof, Theorem 2.2 is derived from Remark 2.4 and the restriction $d \geq 2$ comes up in the transition from (3) to (2). With $d=1$, the correct SDE for $v_{k}$ is expected to be more complicated than (2), much like the SDE for the absolute value of a one-dimensional Brownian motion.

\section{Proof of Theorem 2.2}

\subsection{Strategy}

We split the velocities $p_{k}$ into "slow" and "fast" components, such that the fast component is independent from the slow one. Then we write the evolution of the slow component as a rough differential equation, where the noise is generated by the fast component alone. We show that the noise, suitably rescaled, converges as $\varepsilon \rightarrow 0$ to an enhanced Brownian motion in a suitable rough path topology. Then we use the Itô-Lyons continuity theorem to describe the limiting slow process. Finally, we recover the speeds $v_{k}$ from the slow process.

\subsection{Decomposition into fast and slow components}

We start by (re)defining the random collisions in a convenient way. For $1 \leq k \leq N$, let $\tau_{k}=$ $\left(\tau_{k}^{1}, \tau_{k}^{2}, \ldots\right)$ be Poisson processes with rate $\lambda$, and let $g_{k}^{1}, g_{k}^{2}, \ldots$ be random matrices in the orthogonal group $O(d)$, chosen uniformly (i.e. with respect to the Haar measure). We assume that all these are mutually independent.

We make the $k$-th particle collide at times $\tau_{k}^{n}$, with the instantaneous change of velocity at time $\tau_{k}^{n}$ given by $p_{k}\left(\tau_{k}^{n}\right)=g_{k}^{n} p_{k}\left(\tau_{k}^{n}-0\right)$, where $p_{k}(t-0)$ stands for the left limit of $p_{k}$ at time $t$.

Define $\phi_{k}:[0, \infty) \rightarrow O(d), k=1 \ldots N$ by $\phi_{k}(t)=I$ for $t<\tau_{k}^{1}$ and

$$
\phi_{k}(t)=g_{k}^{n} g_{k}^{n-1} \cdots g_{k}^{1} \quad \text { for } \quad t \in\left[\tau_{k}^{n}, \tau_{k}^{n+1}\right), \quad n \geq 1
$$

Let $u_{k}=\phi_{k}^{*} p_{k}$, where the star means the transpose (also inverse in $O(d)$ ). Then $u_{k}(0)=p_{k}(0)$ and $\left|u_{k}\right|=\left|p_{k}\right|=v_{k}$ at all times. Observe that $u_{k}$ does not jump at collisions, in contrast with $p_{k}$. From the equations of motion (1) we obtain

$$
d u_{k}=\left(\phi_{k}^{*} E-u_{k} \frac{\sum_{j} u_{j}^{*} \phi_{j}^{*} E}{U}\right) d t .
$$

Note that $u_{k}$ are continuous and piecewise smooth processes.

Let $\Phi_{k}(t)=\int_{0}^{t} \phi_{k}^{*}(s) \hat{n} d s$. Writing all $u_{k}, 1 \leq k \leq N$ as one $N d$-dimensional vector $u$, and similarly all $\Phi_{k}$ as one vector $\Phi$, we rewrite (4) as

$$
d u=\varepsilon A(u) d \Phi
$$

where $A(u)=I-\frac{u u^{*}}{U}$ is a smooth matrix valued function.

Define $u^{\varepsilon}(t)=u\left(\varepsilon^{-2} t\right)$ and $W^{\varepsilon}(t)=\varepsilon \Phi\left(\varepsilon^{-2} t\right)$. For every $\varepsilon>0$, the process $W^{\varepsilon}$ is piecewise smooth and $u^{\varepsilon}$ is the solution of an ordinary differential equation

$$
d u^{\varepsilon}=A\left(u^{\varepsilon}\right) d W^{\varepsilon}, \quad u^{\varepsilon}(0)=\xi
$$

where $\xi \in \mathbb{R}^{N d}$ corresponds to the initial condition $u_{k}^{\varepsilon}(0)=p_{k}(0)$.

The above is the decomposition into a "slow" component $u$ and an uncoupled "fast" component $\Phi$. While $u$ has no obvious physical meaning, we can still use it to recover the speeds, because $v_{k}=\left|u_{k}\right|$. 


\subsection{Limiting behavior}

Our next goal is to identify the limit of $u^{\varepsilon}$ as $\varepsilon \rightarrow 0$. We can treat $u^{\varepsilon}$ as a function of $W^{\varepsilon}$. Indeed, there is a solution map $\Gamma$, defined on all piecewise smooth paths, such that $\Gamma\left(W^{\varepsilon}\right)=u^{\varepsilon}$ for each $\varepsilon>0$.

We will show that $W^{\varepsilon}$ converges to a $d N$-dimensional Brownian motion $W$ with covariance matrix $2 \lambda^{-1} d^{-1} I$. (Convergence is weak in the $C^{0}\left([0, \infty), \mathbb{R}^{d}\right)$ topology.)

Then, heuristically, one would expect that $u^{0}=\lim _{\varepsilon \rightarrow 0} \Gamma\left(W^{\varepsilon}\right)=\Gamma(W)$. Such a statement requires continuity of $\Gamma$ on a suitable space of paths, including all $W^{\varepsilon}$ and $W$. There are two immediate problems:

- As a Brownian motion, $W$ is rather irregular, so $\Gamma(W)$ cannot be understood as a solution of an ordinary differential equation. It needs an interpretation, possibly as a solution of the stochastic differential equation $d u^{0}=A\left(u^{0}\right) \diamond d W$, where $\diamond$ means integration in the sense of e.g. Itô, Stratonovich or backward Itô.

- There is no reason for $\Gamma$ to be continuous. In fact, no matter what interpretation of $\Gamma(W)$ we choose, $\Gamma$ will fail to be continuous in any usable way. (We provide a standard example in Appendix $\mathrm{A}$,

Identifying $u^{0}$ is a standard problem in the theory of rough paths. We fix $T>0$ and consider all processes in the time interval $[0, T]$. For $s, t \in[0, T]$, let

$$
W^{\varepsilon}(s, t)=W^{\varepsilon}(t)-W^{\varepsilon}(s), \quad \quad \mathbb{W}^{\varepsilon}(s, t)=\int_{s}^{t} W^{\varepsilon}(r) \otimes d W^{\varepsilon}(r) .
$$

Here $\otimes$ denotes the tensor product, i.e. if $A, B \in \mathbb{R}^{n}$, then $A \otimes B \in \mathbb{R}^{n \times n}$ is given by $(A \otimes B)_{j, k}=$ $A_{j} B_{k}$.

The pairs $\left(W^{\varepsilon}, \mathbb{W}^{\varepsilon}\right)$ are the canonical lifts of the original piecewise smooth paths $W^{\varepsilon}$, see [12, Section 2]. These pairs belong to the space $\mathcal{C}^{\alpha}$ of $\alpha$-Hölder rough paths with $\alpha \in(1 / 3,1 / 2)$.

Fix $\alpha \in(1 / 3,1 / 2)$. The space $\mathcal{C}^{\alpha}$ consists of pairs $(X, \mathbb{X}) \in C^{0}\left([0, T] \times[0, T], \mathbb{R}^{N d} \times \mathbb{R}^{N d \times N d}\right)$ with

$$
\|(X, \mathbb{X})\|_{\alpha}=|X|_{\alpha}+\sqrt{|\mathbb{X}|_{2 \alpha}}<\infty
$$

where

$$
|X|_{\alpha}=\sup _{s \neq t \in[0, T]} \frac{|X(s, t)|}{|t-s|^{\alpha}} \quad \text { and } \quad|\mathbb{X}|_{2 \alpha}=\sup _{s \neq t \in[0, T]} \frac{|\mathbb{X}(s, t)|}{|t-s|^{2 \alpha}}
$$

Further we require that $(X, \mathbb{X})$ satisfies $X(t, t)=0$ and $\mathbb{X}(t, t)=0$ for all $t$, and

$$
\mathbb{X}_{s, t}-\mathbb{X}_{s, u}-\mathbb{X}_{u, t}=X_{s, u} \otimes X_{u, t}
$$

The above is known as the "Chen's relation". It holds for and is inspired by the canonical lifts of smooth paths such as $\left(W^{\varepsilon}, \mathbb{W}^{\varepsilon}\right)$. Because of the Chen's relation, $\mathcal{C}^{\alpha}$ is not a linear subspace of $C^{0}$.

The topology on $\mathcal{C}^{\alpha}$ is inherited from the seminorm $\|\cdot\|_{\alpha}$ on $C^{0}$ : it is given by the distance

$$
d_{\mathcal{C}^{\alpha}}((X, \mathbb{X}),(Y, \mathbb{Y}))=\sup _{s \neq t \in[0, T]} \frac{|X(s, t)-Y(s, t)|}{|t-s|^{\alpha}}+\sup _{s \neq t \in[0, T]} \frac{|\mathbb{X}(s, t)-\mathbb{Y}(s, t)|}{|t-s|^{2 \alpha}}
$$

A key result in rough paths is the Itô-Lyons continuity theorem [12, Section 8]. Applied to the differential equation ([6), it gives a continuous map $\Gamma: \mathcal{C}^{\alpha} \rightarrow C^{0}\left([0, T], \mathbb{R}^{N d}\right)$, such that $\Gamma\left(W^{\varepsilon}, \mathbb{W}^{\varepsilon}\right)=u^{\varepsilon}$ for all $\varepsilon$. 
Remark 3.1. Our application of the Itô-Lyons continuity theorem requires that the function $A(u)$ in (6) has bounded continuous derivatives up to the second order. Our function $A(u)=I-\frac{u u^{*}}{U}$ is not bounded on $\mathbb{R}^{N d}$. However, all the solutions $u^{\varepsilon}$ are uniformly bounded, namely restricted to the sphere $\left|u^{\varepsilon}\right|^{2}=U$, where the global unboundedness of $A(u)$ or its derivatives does not cause problems.

Suppose now that the random elements $\left(W^{\varepsilon}, \mathbb{W}^{\varepsilon}\right)$ converge weakly in $\mathcal{C}^{\alpha}$ to some $(W, \mathbb{W})$. Then, using the continuous mapping theorem, we find $u^{0}=\Gamma(W, \mathbb{W})$.

Further, in Section 4, we show that $\left(W^{\varepsilon}, \mathbb{W}^{\varepsilon}\right)$ do indeed converge to a $\mathcal{C}^{\alpha}$-valued random process $(W, \mathbb{W})$, where $W$ is the Brownian motion with covariance matrix $2 \lambda^{-1} d^{-1} I$ and, using odW to denote Stratonovich integration,

$$
W(s, t)=W(t)-W(s), \quad \mathbb{W}(s, t)=\int_{s}^{t} W(r) \otimes \circ d W(r) .
$$

Remark 3.2. A rough path $(W, \mathbb{W})$, where $W$ is a Brownian motion, is often referred to as enhanced Brownian motion. In our case, the enhancement is Stratonovich. Often it is natural to consider the Itô enhancement; in general, the options for enhancement are plentiful.

Rough integration against $(W, \mathbb{W})$ coincides with Stratonovich integration against $W$ (see [12]). This means that $u^{0}=\Gamma(W, \mathbb{W})$ is the solution of the Stratonovich stochastic differential equation $d u^{0}=A\left(u^{0}\right) \circ d W$. The convergence is on the time interval $[0, T]$, but $T$ is arbitrary, so convergence on $[0, \infty)$ follows.

In other words, we obtain the following result:

Theorem 3.3. As $\varepsilon \rightarrow 0$, the processes $u^{\varepsilon}$ converge weakly in the $C^{0}\left([0, \infty), \mathbb{R}^{N d}\right)$ topology to $u^{0}$, the solution of Stratonovich differential equation

$$
d u^{0}=\left(I-\frac{u^{0}\left(u^{0}\right)^{*}}{U}\right) \circ d W, \quad u^{0}(0)=\xi
$$

Here $W$ is a dN-dimensional Brownian motion with covariance matrix $2 \lambda^{-1} d^{-1} I$.

Remark 3.4. The process (7) is a diffusion on the sphere $\left|u^{0}\right|^{2}=U$. When $N d$ is large and $U=N d$, coordinate projections of $u^{0}$ are close to an Ornstein-Uhnelbeck process. We provide the details in Appendix B.

Remark 3.5. Theorem 2.2 is a corollary of Theorem 3.3 .

\section{Convergence of rough paths}

In Section 3 we introduced the random rough paths $\left(W^{\varepsilon}, \mathbb{W}^{\varepsilon}\right)$ and $(W, \mathbb{W})$. To prove Theorem 3.3 , it remains to show that $\left(W^{\varepsilon}, \mathbb{W}^{\varepsilon}\right)$ converge weakly to $(W, \mathbb{W})$ in the space $\mathcal{C}^{\alpha}$ of $\alpha$-Hölder rough paths, with $\alpha \in(1 / 3,1 / 2)$. This is the goal of this section.

We follow the arguments for deterministic dynamical systems by Kelly and Melbourne [13]. Our situation is simpler because of randomness, but [13] does not cover it, so we provide an adaption of their proof.

Remark 4.1. Writing $W^{\varepsilon}(t)=W^{\varepsilon}(0, t)$ and $\mathbb{W}^{\varepsilon}(t)=\mathbb{W}^{\varepsilon}(0, t)$, we can work with the rough paths $\left(W^{\varepsilon}, \mathbb{W}^{\varepsilon}\right)$ as a random processes, defined on $t \in[0, \infty)$. The "increments" $W^{\varepsilon}(s, t)$ and $\mathbb{W}^{\varepsilon}(s, t)$ can be recovered from the Chen's relation:

$$
\begin{aligned}
& W^{\varepsilon}(s, t)=W^{\varepsilon}(t)-W^{\varepsilon}(s), \\
& \mathbb{W}^{\varepsilon}(s, t)=\mathbb{W}^{\varepsilon}(t)-\mathbb{W}^{\varepsilon}(s)-W^{\varepsilon}(s) \otimes W^{\varepsilon}(t) .
\end{aligned}
$$


To prove convergence of $\left(W^{\varepsilon}, \mathbb{W}^{\varepsilon}\right)$ to $(W, \mathbb{W})$ in the $\mathcal{C}^{\alpha}$ topology, it is sufficient to show convergence in the weaker uniform topology together with suitable moment bounds (c.f. [13, Theorem 9.1]):

Lemma 4.2. As $\varepsilon$ goes to zero, the process $\left(W^{\varepsilon}, \mathbb{W}^{\varepsilon}\right)$ converges weakly to $(W, \mathbb{W})$ in $C^{0}\left([0, \infty), \mathbb{R}^{N d} \times \mathbb{R}^{N d \times N d}\right)$.

Moreover, there exist $q>3$ and $C>0$ such that for all $\varepsilon>0$ and $0 \leq s \leq t$,

$$
\left\|W^{\varepsilon}(s, t)\right\|_{2 q} \leq C|t-s|^{1 / 2} \quad \text { and } \quad\left\|\mathbb{W}^{\varepsilon}(s, t)\right\|_{q} \leq C|t-s| .
$$

Here $\|X\|_{q}=\left(\mathbb{E}|X|^{q}\right)^{1 / q}$ is the $L^{q}$ norm.

Here is an outline of the proof of Lemma 4.2 ;

- Observe that the processes $W^{\varepsilon}(t)$ do not have stationary increments. This comes from the fact that $\phi_{k}(0)=I$ instead of being distributed uniformly in $O(d)$. This is a minor inconvenience which we solve by introducing a random time shift $\tau$ such that the random processes $\hat{W}^{\varepsilon}(t)=W^{\varepsilon}\left(\varepsilon^{2} \tau+t\right)-W^{\varepsilon}\left(\varepsilon^{2} \tau\right)$ do have stationary increments. We show that the rough paths $\left(\hat{W}^{\varepsilon}, \hat{\mathbb{W}}^{\varepsilon}\right)$, where $\hat{\mathbb{W}}^{\varepsilon}$ are the respective iterated integrals, well approximate $\left(W^{\varepsilon}, \mathbb{W}^{\varepsilon}\right)$.

- We represent $\hat{W}^{\varepsilon}(t)=\varepsilon \int_{0}^{\varepsilon^{-2} t} h \circ F^{s} d s$, where $F^{t}: \Omega \rightarrow \Omega$ is a measure preserving semiflow on a probability space $(\Omega, \mathbb{P})$ and $h: \Omega \rightarrow \mathbb{R}^{N d}$ is an observable.

- We consider a discrete time dynamical system $F: \Omega \rightarrow \Omega$, where $F=F^{1}$. It preserves the measure $\mathbb{P}$, and our construction ensures that $F$ is mixing. The semiflow $F^{t}$ is a suspension over $F$ with the roof function equal to 1 . We consider the induced observable $V: \Omega \rightarrow \mathbb{R}^{N d}$, $V=\int_{0}^{1} h \circ F^{t} d t$, and use a martingale-coboundary decomposition $V=m+\chi \circ F-\chi$, where both $\chi$ and $m$ are bounded and $m$ is a "martingale part". This means that for every $n$, the "backward" sum $\sum_{j=n-k}^{n} m \circ F^{j}$ is a martingale on $k=0, \ldots, n$.

- Let $\widetilde{W}^{\varepsilon}(t)=\varepsilon \sum_{j=0}^{\left.\varepsilon^{-2} t\right\rfloor} V \circ F^{j}$ be a discrete time version of $\hat{W}$, and let $\widetilde{\mathbb{W}} \varepsilon$ be the corresponding iterated integral. Then $(\widetilde{W}, \widetilde{\mathbb{W}})$ is a random càdlàg process. By [13, Theorem 4.3], if $F$ is mixing and $V$ allows a martingale-coboundary decomposition as above, then the weak limit of $\left(\widetilde{W}^{\varepsilon}, \widetilde{\mathbb{W}}^{\varepsilon}\right)$ in the $C^{0}$ topology is described by Green-Kubo-like formulas (10).

- The processes $\left(\widetilde{W^{\varepsilon}}, \widetilde{\mathbb{W}^{\varepsilon}}\right),\left(\hat{W}^{\varepsilon}, \widehat{\mathbb{W}}^{\varepsilon}\right)$ and $\left(W^{\varepsilon}, \mathbb{W}^{\varepsilon}\right)$ are closely related, and knowing the weak limit of the first allows us to compute the weak limit of the others.

- The moment bounds for $\left(\widetilde{W^{\varepsilon}}, \widetilde{\mathbb{W}^{\varepsilon}}\right)$ and $\left(\hat{W}^{\varepsilon}, \widehat{\mathbb{W}}^{\varepsilon}\right)$, and hence for $\left(W^{\varepsilon}, \mathbb{W}^{\varepsilon}\right)$, are implications of the martingale-coboundary decomposition [13, Section 7].

In the rest of this section, we implement the above.

\subsection{Probability measure preserving flow}

Note that the processes $\phi_{k}$ are not stationary. For instance, $\phi_{k}(0)=I$ for all $k$. Let $\tau=$ $\max \left\{\tau_{k}^{1}: 1 \leq k \leq N\right\}$ and $\hat{\phi}_{k}(t)=\phi_{k}(t+\tau), t \geq 0$. Now, $\hat{\phi}_{k}$ are stationary processes, and so are $\hat{\phi}_{k}^{*} \hat{n}$. 
Let $\psi$ be a random process with values in $\mathbb{R}^{N d}$, obtained by stacking together all the coordinates of $\hat{\phi}_{k}^{*} \hat{n}, k=1 \ldots N$.

Let $\Omega=D\left([0, \infty), \mathbb{R}^{N d}\right)$ be the space of càdlàg functions.

Let $\mathbb{P}$ be the probability measure on $\Omega$, corresponding to the distribution of $\psi$, and let $\mathbb{E}$ denote the corresponding expectation.

Define the flow $F^{t}: \Omega \rightarrow \Omega$ by $\left(F^{t} x\right)(s)=x(t+s)$ for $s, t \geq 0$, and let $h: \Omega \rightarrow \mathbb{R}^{N d}, h(x)=x(0)$ be an observable. Since $\psi$ is a stationary process, the measure $\mathbb{P}$ is $F^{t}$-invariant.

Define $\hat{W}^{\varepsilon}$ and $\hat{\mathbb{W}}^{\varepsilon}$ by

$$
\hat{W}^{\varepsilon}(t)=\varepsilon \int_{0}^{\varepsilon^{-2} t} h \circ F^{s} d s \quad \text { and } \quad \hat{\mathbb{W}}^{\varepsilon}(t)=\int_{0}^{t} \hat{W}^{\varepsilon}(s) \otimes d \hat{W}^{\varepsilon}(s) .
$$

Remark 4.3. Where it is convenient, we assume that $W^{\varepsilon}, \hat{W}^{\varepsilon}$ and $\tau$ are defined on the same probability space such that $W^{\varepsilon}\left(\varepsilon^{2} \tau+t\right)-W^{\varepsilon}\left(\varepsilon^{2} \tau\right)=\hat{W}^{\varepsilon}(t)$ for all $t \geq 0$. The iterated integrals $\mathbb{W}^{\varepsilon}$ and $\hat{\mathbb{W}}^{\varepsilon}$ are fully determined by $W^{\varepsilon}$ and $\hat{W}^{\varepsilon}$, so they also belong to this probability space.

\subsection{Discrete time system}

Let $F=F^{1}$, and $P: L^{1}(\Omega) \rightarrow L^{1}(\Omega)$ be the (Ruelle-Perron-Frobenius) transfer operator, corresponding to $F$ and $\mathbb{P}$. Formally, $P$ is defined by

$$
\mathbb{E}(P v w)=\mathbb{E}(v w \circ F) \quad \text { for all } v \in L^{1}(\Omega) \text { and } w \in L^{\infty}(\Omega) .
$$

Remark 4.4. Pv can be computed explicitly. For $x \in \Omega$,

$$
(P v)(x)=\mathbb{E}(v \mid F=x)=\int_{\Omega} v(y) d \mathbb{P}(y \mid F y=x),
$$

where $\mathbb{P}(\cdot \mid F=x)$ is the regular conditional probability corresponding to the observable $F: \Omega \rightarrow$ $\Omega$, and $\mathbb{E}(\cdot \mid F=x)$ is the corresponding expectation. (See [8] for a guide on guilt-free manipulation with regular conditional probabilities.)

Similarly, $\left(P^{k} v\right)(x)=\mathbb{E}\left(v \mid F^{k}=x\right)$ for $k \geq 1$.

Define $V: \Omega \rightarrow \mathbb{R}^{N d}$ by $V=\int_{0}^{1} h \circ F^{t} d t$. Then $V(x)=\int_{0}^{1} x(t) d t$ for $x \in \Omega$. Note that $V$ is a bounded random variable, and due to the natural symmetries of our model, $\mathbb{E} V=0$.

Proposition 4.5. For $a \in \mathbb{R}^{N d}$,

$$
\int_{\Omega} x(t) d \mathbb{P}(x \mid x(0)=a)=e^{-\lambda t} a .
$$

Proof. Recall the definition of $\phi_{k}$. Note that for $a^{\prime} \in O(d)$,

$$
\begin{aligned}
& \mathbb{E}\left(\phi_{k}^{*}(t) \hat{n} \mid \phi_{k}(0)=a^{\prime}, t<\tau_{k}^{1}\right)=a^{\prime *} \hat{n}, \quad \text { and } \\
& \mathbb{E}\left(\phi_{k}^{*}(t) \hat{n} \mid \phi_{k}(0)=a^{\prime}, t \geq \tau_{k}^{1}\right)=0 .
\end{aligned}
$$

Hence

$$
\mathbb{E}\left(\phi_{k}^{*} \hat{n} \mid \phi_{k}(0)=a^{\prime}\right)=\mathbb{P}\left(t<\tau_{k}^{1}\right) a^{\prime *} \hat{n}=e^{-\lambda t} a^{\prime *} \hat{n}
$$

The result follows. 
Proposition 4.6. For $x \in \Omega$ and $k \geq 0$,

$$
\left(P^{k} V\right)(x)=\frac{e^{-\lambda k}\left(e^{\lambda}-1\right)}{\lambda} x(0) .
$$

Proof. We observe that $(\Omega, \mathbb{P})$ is probability space of Markov, stationary and time-reversible processes. In particular, for $x \in \Omega, t \in[0,1]$ and $k \geq 1$,

$$
\begin{aligned}
& \int_{\Omega} y(t) d \mathbb{P}\left(y \mid F^{k} y=x\right)=\int_{\Omega} y(t) d \mathbb{P}(y \mid y(k)=x(0)) \\
& =\int_{\Omega} y(k-t) d \mathbb{P}(y \mid y(0)=x(0))=e^{-\lambda(k-t)} x(0),
\end{aligned}
$$

where in the last step we used Proposition 4.5.

Using (9) and Fubini's theorem, write for $k \geq 1$ :

$$
\begin{aligned}
\left(P^{k} V\right)(x) & =\mathbb{E}\left(V \mid F^{k}=x\right)=\int_{\Omega} \int_{0}^{1} y(t) d t d \mathbb{P}\left(y \mid F^{k} y=x\right) \\
& =\int_{0}^{1} \int_{\Omega} y(t) d \mathbb{P}\left(y \mid F^{k} y=x\right) d t=\int_{0}^{1} e^{-\lambda(k-t)} x(0) d t=\frac{e^{-\lambda k}\left(e^{\lambda}-1\right)}{\lambda} x(0) .
\end{aligned}
$$

Now we approximate $V$ by a martingale, following [13, Section 4]. Define $\chi, m: \Omega \rightarrow \mathbb{R}^{N d}$ by

$$
\chi=\sum_{k=1}^{\infty} P^{k} V \quad \text { and } \quad V=m+\chi \circ F-\chi .
$$

Using Proposition 4.6 and the definition of $V$, we compute $\chi$ and $m$ explicitly:

$$
\chi(x)=\frac{1}{\lambda} x(0) \quad \text { and } \quad m(x)=\int_{0}^{1} x(t) d t+\frac{x(0)-x(1)}{\lambda} .
$$

Clearly $m, V \in L^{\infty}(\Omega)$. It is standard that $P m=0$ (see [13, Proposition 4.4]).

Let $V_{i}$ and $x_{i}$ denote the $i$-th coordinates of $V$ and $x$ respectively.

\section{Proposition 4.7.}

$$
\int_{\Omega} x_{i}(t) x_{j}(s) d \mathbb{P}(x)=\left\{\begin{array}{ll}
0, & i \neq j \\
d^{-1} e^{-\lambda|t-s|}, & i=j
\end{array} .\right.
$$

Proof. Let $r \geq 0$, and let $x$ be distributed in $\Omega$ according to $\mathbb{P}$. Note that $\sum_{j=1}^{N d} x_{j}^{2}(r)=N$. Then due to the symmetry of the distribution $\mathbb{P}$,

$$
\int_{\Omega} x_{j}^{2}(r) d \mathbb{P}(x)=d^{-1} \quad \text { for every } j
$$

Fix $j$. By Proposition 4.5,

$$
\int_{\Omega} x_{j}(0) x_{j}(r) d \mathbb{P}(x \mid x(0))=x_{j}^{2}(0) e^{-\lambda r}
$$


Without loss of generality suppose that $s \leq t$. By the above, and using the fact that the measure $\mathbb{P}$ is stationary, write

$$
\begin{aligned}
\int_{\Omega} x_{j}(t) x_{j}(s) d \mathbb{P}(x) & =\int_{\Omega} x_{j}(0) x_{j}(t-s) d \mathbb{P}(x) \\
& =\int_{\Omega} \int_{\Omega} x_{j}(0) x_{j}(t-s) d \mathbb{P}(x \mid x(0)) d \mathbb{P}(x) \\
& =\int_{\Omega} x_{j}^{2}(0) e^{-\lambda(t-s)} d \mathbb{P}(x)=d^{-1} e^{-\lambda(t-s)} .
\end{aligned}
$$

It remains to show that $\int_{\Omega} x_{i}(t) x_{j}(s) d \mathbb{P}(x)=0$ when $i \neq j$. For this we use again a symmetry of the distribution $\mathbb{P}$ : for each $j$, it is invariant under transformation $x_{j} \mapsto-x_{j}$. Thus with $i \neq j$,

$$
\int_{\Omega} x_{i}(t) x_{j}(s) d \mathbb{P}(x)=-\int_{\Omega} x_{i}(t) x_{j}(s) d \mathbb{P}(x)=0 .
$$

Proposition 4.8. If $i \neq j$, then $\mathbb{E}\left(V_{i} V_{j} \circ F^{k}\right)=0$. Also,

$$
\mathbb{E}\left(V_{i} V_{i} \circ F^{k}\right)=\left\{\begin{array}{ll}
\frac{\left(e^{\lambda}-1\right)^{2} e^{-\lambda(k+1)}}{\lambda^{2} d}, & k \geq 1 \\
\frac{2\left(e^{-\lambda}+\lambda-1\right)}{\lambda^{2} d}, & k=0
\end{array} .\right.
$$

Proof. Use Fubini's theorem and Proposition 4.7.

$$
\begin{aligned}
\mathbb{E}\left(V_{i} V_{j} \circ F^{k}\right) & =\int_{\Omega} \int_{0}^{1} x_{i}(t) d t \int_{k}^{k+1} x_{j}(s) d s d \mathbb{P}(x) \\
& =\int_{0}^{1} \int_{k}^{k+1} \int_{\Omega} x_{i}(t) x_{j}(s) d \mathbb{P}(x) d s d t=\int_{0}^{1} \int_{k}^{k+1} d^{-1} e^{-\lambda|s-t|} d s d t .
\end{aligned}
$$

The result follows.

Define

$$
\begin{aligned}
& \widetilde{E}=\sum_{k=1}^{\infty} \mathbb{E}\left(V \otimes V \circ F^{k}\right), \\
& \widetilde{\Sigma}=\mathbb{E}(V \otimes V)+\sum_{k=1}^{\infty} \mathbb{E}\left(V \otimes V \circ F^{k}+V \circ F^{k} \otimes V\right) .
\end{aligned}
$$

Corollary 4.9. $\widetilde{E}=\frac{1-e^{-\lambda}}{\lambda^{2} d} I$ and $\widetilde{\Sigma}=\frac{2}{\lambda d} I$.

Define a càdlàg process $\left(\widetilde{W^{\varepsilon}}, \widetilde{\mathbb{W}^{\varepsilon}}\right): \Omega \rightarrow D\left([0, \infty), \mathbb{R}^{N d} \times \mathbb{R}^{N d \times N d}\right)$ by

$$
\widetilde{W^{\varepsilon}}(t)=\varepsilon \sum_{j=0}^{\left\lfloor\varepsilon^{-2} t\right\rfloor} V \circ F^{j} \quad \text { and } \quad \widetilde{\mathbb{W}}^{\varepsilon}(t)=\int_{0}^{t} \widetilde{W}^{\varepsilon}(s) \otimes d \widetilde{W}^{\varepsilon}(s) .
$$

By [13, Theorem 4.3], $\left(\widetilde{W^{\varepsilon}}, \widetilde{\mathbb{W}}^{\varepsilon}\right)$ converges weakly to $(\widetilde{W}, \widetilde{\mathbb{W}})$ in $D\left([0, \infty), \mathbb{R}^{N d} \times \mathbb{R}^{N d \times N d}\right)$ in the uniform topology, where $\widetilde{W}$ is the $N$-dimensional Brownian motion with covariance matrix $\widetilde{\Sigma}$, and

$$
\widetilde{\mathbb{W}}(t)=\int_{0}^{t} \widetilde{W} \otimes d \widetilde{W}+\widetilde{E} t
$$




\subsection{Continuous time system}

In this part of the proof we are closely following [13, Section 6].

Define $H: \Omega \times[0,1) \rightarrow \mathbb{R}^{N d}, H(x, r)=\int_{0}^{r} x(t) d t$. Let

$$
E=\widetilde{E}+\int_{\Omega} \int_{0}^{1} H(x, r) \otimes x(r) d r d \mathbb{P}(x) .
$$

Proposition 4.10. $E=\frac{1}{\lambda d} I$.

Proof. Indeed, using the definition of $H$, Fubini's theorem and Proposition 4.7, write

$$
\begin{aligned}
& \int_{\Omega} \int_{0}^{1} H(x, r) \otimes x(r) d r d \mathbb{P}(x)=\int_{\Omega} \int_{0}^{1} \int_{0}^{r} x(t) d t \otimes x(r) d r d \mathbb{P}(x) \\
& =\int_{0}^{1} \int_{0}^{r} \int_{\Omega} x(t) \otimes x(r) d \mathbb{P}(x) d t d r=\int_{0}^{1} \int_{0}^{r} d^{-1} e^{-\lambda|t-r|} I d t d r=\frac{e^{-\lambda}+\lambda-1}{\lambda^{2} d} I .
\end{aligned}
$$

The result follows from Corollary 4.9,

Recall the definition of $\hat{W}^{\varepsilon}$ and $\hat{\mathbb{W}}^{\varepsilon}$. By [13, Theorem 6.1], $\left(\hat{W}^{\varepsilon}, \hat{\mathbb{W}}^{\varepsilon}\right)$ converges weakly to $(W, \mathbb{W})$ in $C^{0}\left([0, \infty), \mathbb{R}^{N d} \times \mathbb{R}^{N d \times N d}\right)$, where $W$ is the $N$-dimensional Brownian motion with covariance

matrix $\Sigma=\widetilde{\Sigma}$, and $\mathbb{W}(t)=\int_{0}^{t} W \otimes d W+E t$. Converting the Itô integral to Stratonovich, we obtain

$$
\mathbb{W}(t)=\int_{0}^{t} W \otimes \circ d W-\frac{1}{2} \Sigma t+E t=\int_{0}^{t} W \otimes \circ d W .
$$

We modelled the flow $F^{t}$ as a suspension over $F$ with the roof function identically equal to 1 . Both $h$ and $V$ are bounded observables, and we have the $L^{\infty}$ martingale-coboundary decomposition $V=m+\chi \circ F-\chi$. Therefore the results of [13, Subsection 7.2] apply. By [13, Corollary 7.6], for every $q>3$ there exists $C>0$ such that for all $0 \leq s \leq t$,

$$
\left\|\hat{W}^{\varepsilon}(s, t)\right\|_{2 q} \leq C|t-s|^{1 / 2} \quad \text { and } \quad\left\|\hat{\mathbb{W}}^{\varepsilon}(s, t)\right\|_{q} \leq C|t-s| .
$$

\subsection{Completion of the proof of Lemma 4.2}

We proved Lemma 4.2 for the processes $\left(\hat{W}^{\varepsilon}, \hat{\mathbb{W}}^{\varepsilon}\right)$ in place of $\left(W^{\varepsilon}, \mathbb{W}^{\varepsilon}\right)$. As in Remark 4.3 , the two are related by the time shift

$$
\hat{W}^{\varepsilon}(t)=W^{\varepsilon}\left(\varepsilon^{2} \tau, \varepsilon^{2} \tau+t\right) \quad \text { and } \quad \hat{\mathbb{W}}^{\varepsilon}(t)=\mathbb{W}^{\varepsilon}\left(\varepsilon^{2} \tau, \varepsilon^{2} \tau+t\right)
$$

for all $t \geq 0$.

It remains to prove the moment bounds (8), based on (11), and to show that $\left(\hat{W}^{\varepsilon}, \hat{\mathbb{W}}^{\varepsilon}\right)$ and $\left(W^{\varepsilon}, \mathbb{W}^{\varepsilon}\right)$ are close in $C^{0}\left([0, \infty), \mathbb{R}^{N d} \times \mathbb{R}^{N d \times N d}\right)$. This is done in the following two propositions.

Proposition 4.11. For every $q>3$ there exists $C>0$ such that for all $0 \leq s \leq t$,

$$
\begin{gathered}
\left\|W^{\varepsilon}(s, t)\right\|_{2 q} \leq C|t-s|^{1 / 2} \\
\left\|\mathbb{W}^{\varepsilon}(s, t)\right\|_{q} \leq C|t-s|
\end{gathered}
$$

Proof. As in Remark 4.3, we assume that $\tau, W^{\varepsilon}$ and $\hat{W}^{\varepsilon}$ are defined on the same probability space such that $W^{\varepsilon}\left(\varepsilon^{2} \tau+t\right)-W^{\varepsilon}\left(\varepsilon^{2} \tau\right)=\hat{W}^{\varepsilon}(t)$ for all $t \geq 0$, and $\hat{W}^{\varepsilon}$ is independent from $\tau$.

Note that $\|\tau\|_{q}$ is finite for every $q \geq 1$.

Assume that $0 \leq s \leq t$. First we show (12). We consider three cases: 
(a) If $s \leq t \leq \varepsilon^{2} \tau$, then $\left|W^{\varepsilon}(s, t)\right| \ll \varepsilon^{-1}|t-s| \ll \tau^{1 / 2}|t-s|^{1 / 2}$, thus

$$
\left\|W^{\varepsilon}(s, t) 1_{s \leq t \leq \varepsilon^{2} \tau}\right\|_{2 q} \ll|t-s|^{1 / 2} .
$$

(b) If $\varepsilon^{2} \tau \leq s \leq t$, then $W^{\varepsilon}(s, t)=\hat{W}^{\varepsilon}\left(s-\varepsilon^{2} \tau, t-\varepsilon^{2} \tau\right)$, and by (11),

$$
\left\|W^{\varepsilon}(s, t) 1_{\varepsilon^{2} \tau \leq s \leq t}\right\|_{2 q} \ll|t-s|^{1 / 2} .
$$

(c) If $s \leq \varepsilon^{2} \tau \leq t$, then $W^{\varepsilon}(s, t)=W^{\varepsilon}\left(s, \varepsilon^{2} \tau\right)+W^{\varepsilon}\left(\varepsilon^{2} \tau, t\right)$ and by (a) and (b),

$$
\left\|W^{\varepsilon}(s, t) 1_{s \leq \varepsilon^{2} \tau \leq t}\right\|_{2 q} \leq\left\|W^{\varepsilon}\left(s, \varepsilon^{2} \tau\right) 1_{s \leq \varepsilon^{2} \tau \leq t}\right\|_{2 q}+\left\|W^{\varepsilon}\left(\varepsilon^{2} \tau, t\right) 1_{s \leq \varepsilon^{2} \tau \leq t}\right\|_{2 q} \ll|t-s|^{1 / 2} .
$$

The bound (12) follows from the above.

The bound (13) is proved similarly, we consider the same three cases:

(a) Suppose that $s \leq t \leq \varepsilon^{2} \tau$. Observe that the variation of $W^{\varepsilon}$ on the interval $(t, s)$ is $O\left(\varepsilon^{-1} \mid t-\right.$ $s \mid)$, and $\left|W^{\varepsilon}(s, r)\right| \ll \varepsilon^{-1}|t-s|$ for $s \leq r \leq t$. Then

$$
\left|\mathbb{W}^{\varepsilon}(s, t)\right|=\left|\int_{s}^{t} W^{\varepsilon}(s, r) \otimes d W^{\varepsilon}(r)\right| \ll \varepsilon^{-2}|t-s|^{2} \leq \tau|t-s| .
$$

Thus $\left\|\mathbb{W}^{\varepsilon}(s, t) 1_{s \leq t \leq \varepsilon^{2} \tau}\right\|_{q} \ll\|\tau\|_{q}|t-s| \ll|t-s|$.

(b) If $\varepsilon^{2} \tau \leq s \leq t$, then $\mathbb{W}^{\varepsilon}(s, t)=\mathbb{W}^{\varepsilon}\left(s-\varepsilon^{2} \tau, t-\varepsilon^{2} \tau\right)$, so $\left\|\mathbb{W}^{\varepsilon}(s, t) 1_{\varepsilon^{2} \tau \leq s \leq t}\right\|_{q} \ll|t-s|$ by (11).

(c) Suppose that $s \leq \varepsilon^{2} \tau \leq t$. It is convenient to use Chen's relation

$$
\mathbb{W}^{\varepsilon}(s, t)-\mathbb{W}^{\varepsilon}\left(s, \varepsilon^{2} \tau\right)-\mathbb{W}^{\varepsilon}\left(\varepsilon^{2} \tau, t\right)=W^{\varepsilon}\left(s, \varepsilon^{2} \tau\right) \otimes W^{\varepsilon}\left(\varepsilon^{2} \tau, t\right) .
$$

Estimate

$$
\begin{aligned}
\left\|\mathbb{W}^{\varepsilon}\left(s, \varepsilon^{2} \tau\right) 1_{s \leq \varepsilon^{2} \tau \leq t}\right\|_{q} \ll|t-s| & \text { by }(\mathrm{a}), \\
\left\|\mathbb{W}^{\varepsilon}\left(\varepsilon^{2} \tau, t\right) 1_{s \leq \varepsilon^{2} \tau \leq t}\right\|_{q} \ll|t-s| & \text { by (b), } \\
\left\|W^{\varepsilon}\left(s, \varepsilon^{2} \tau\right) \otimes W^{\varepsilon}\left(\varepsilon^{2} \tau, t\right) 1_{s \leq \varepsilon^{2} \tau \leq t}\right\|_{q} \ll|t-s| & \text { by Hölder's inequality and (12). }
\end{aligned}
$$

The bound $\left\|\mathbb{W}^{\varepsilon}(s, t) 1_{s \leq \varepsilon^{2} \tau \leq t}\right\|_{q} \ll|t-s|$ follows.

The relation (13) follows. The proof is complete.

Proposition 4.12. There exists $C>0$ such that for all $t \geq 0$,

(a) $\left|W^{\varepsilon}(t)-\hat{W}^{\varepsilon}(t)\right| \leq C \varepsilon \tau$,

(b) $\left|\mathbb{W}^{\varepsilon}(t)-\hat{\mathbb{W}}^{\varepsilon}(t)\right| \leq C \varepsilon^{2} \tau^{2}$.

As a consequence, the processes $\left(W^{\varepsilon}, \mathbb{W}^{\varepsilon}\right)$ and $\left(\hat{W}^{\varepsilon}, \hat{\mathbb{W}}^{\varepsilon}\right)$ converge to the same limit in $C^{0}$. 
Proof. By construction of $W^{\varepsilon}(s, t)$ and $\mathbb{W}^{\varepsilon}(s, t)$, for all $0 \leq s \leq t$,

$$
\left|W^{\varepsilon}(s, t)\right| \ll \varepsilon^{-1}|t-s| \quad \text { and } \quad\left|\mathbb{W}^{\varepsilon}(s, t)\right| \ll \varepsilon^{-2}|t-s|^{2} .
$$

Similar bounds hold for $\hat{W}^{\varepsilon}$ and $\hat{\mathbb{W}}^{\varepsilon}$. The result for $t \leq \varepsilon^{2} \tau$ follows directly from the above. Suppose that $t \geq \varepsilon^{2} \tau$. Then

$$
\left|W^{\varepsilon}\left(t+\varepsilon^{2} \tau\right)-\hat{W}^{\varepsilon}(t)\right|=\left|W^{\varepsilon}\left(\varepsilon^{2} \tau\right)\right| \ll \varepsilon \tau .
$$

By Chen's relation,

$$
\left|\mathbb{W}^{\varepsilon}\left(t+\varepsilon^{2} \tau\right)-\hat{\mathbb{W}}^{\varepsilon}(t)\right|=\left|\mathbb{W}^{\varepsilon}\left(\varepsilon^{2} \tau\right)+W^{\varepsilon}\left(\varepsilon^{2} \tau\right) \otimes W^{\varepsilon}\left(\varepsilon^{2} \tau, t+\varepsilon^{2} \tau\right)\right| \ll \varepsilon^{2} \tau^{2}
$$

Similarly one shows that

$$
\left|W^{\varepsilon}(t)-W^{\varepsilon}\left(t+\varepsilon^{2} \tau\right)\right| \ll \varepsilon \tau \quad \text { and } \quad\left|\mathbb{W}^{\varepsilon}(t)-\mathbb{W}^{\varepsilon}\left(t+\varepsilon^{2} \tau\right)\right| \ll \varepsilon^{2} \tau^{2}
$$

The result follows.

\section{A Heuristic Analysis}

In this section we give a heuristic derivation of our result based on studying directly the distribution function. This derivation works also for more general collision models, see [5]. To extend the present result to those models requires the analysis with noise that is not independent from the slow variables. This is still a vastly unexplored area.

As before, we consider only the distribution of velocities, which is independent of the positions, and the electric field is $E=\varepsilon \hat{n}$. Let $p=\left(p_{1}, \ldots, p_{N}\right)$ denote velocities of the $N$ particles and let $F_{t}^{\varepsilon}(p)$ be the density of the velocity distribution at time $t$. From (1) we get

$$
\begin{aligned}
\partial_{t} F_{t}^{\varepsilon}(p) & +\sum_{i} \nabla_{p_{i}}\left(\left[E-\frac{\sum_{k} E \cdot p_{k}}{U} p_{i}\right] F_{t}^{\varepsilon}(p)\right) \\
& =\lambda \sum_{i} \int_{S^{d-1}}\left(F_{t}^{\varepsilon}\left(p_{1}, \ldots,\left|p_{i}\right| \omega, \ldots, p_{N}\right)-F_{t}^{\varepsilon}(p)\right) d \sigma(\omega),
\end{aligned}
$$

where $\sigma(\omega)$ is the normalized volume measure on $S^{d-1}$. We write the above equation as

$$
\partial_{t} F_{t}^{\varepsilon}+\varepsilon \mathcal{B} F_{t}^{\varepsilon}=\mathcal{A} F_{t}^{\varepsilon}
$$

Rescaling time as $\widetilde{F}_{t}^{\varepsilon}(v)=F_{t / \varepsilon^{2}}^{\varepsilon}(v)$ and assuming that

$$
\widetilde{F}_{t}^{\varepsilon}(p)=\widetilde{F}_{t}^{0}(p)+\varepsilon \widetilde{F}_{t}^{(1)}(p)+\varepsilon^{2} \widetilde{F}_{t}^{(2)}(p)+o\left(\varepsilon^{2}\right)
$$

we get, collecting the coefficients of powers of $\varepsilon$,

$$
\begin{aligned}
0 & =\mathcal{A} \widetilde{F}_{t}^{0} \\
\mathcal{B} \widetilde{F}_{t}^{0} & =\mathcal{A} \widetilde{F}_{t}^{(1)} \\
\dot{\dot{F}_{t}^{0}-\mathcal{B} \widetilde{F}_{t}^{(1)}} & =\mathcal{A} \widetilde{F}_{t}^{(2)}
\end{aligned}
$$


where the dot indicates differentiation with respect to $t$. From (14) it follows that $\widetilde{F}_{t}^{0}$ depends only on $v_{k}$ while substituting (15) into (16) gives

$$
\dot{\widetilde{F}}_{t}^{0}=P^{\perp} \mathcal{B} \mathcal{A}^{-1} \mathcal{B} \widetilde{F}_{t}^{0},
$$

where $P^{\perp}$ is the orthogonal projection from $L^{2}\left(S^{N d-1}\right)$ to the kernel $H_{0}$ of $\mathcal{A}$. Observe that $\mathcal{A}^{-1} \mathcal{B}$ is well defined: the image of $\mathcal{B}$ is contained in $H_{0}^{\perp}$. Writing (17) explicitly gives

$$
\dot{\vec{F}}_{t}^{0}(v)=-\delta \sum_{i} \frac{\partial}{\partial v_{i}}\left(\left[\frac{d-1}{2 v_{i}}-\frac{(N d-1) v_{i}}{U}\right] \widetilde{F}_{t}^{0}(v)\right)+\frac{\delta}{2} \sum_{i, j} \frac{\partial^{2}}{\partial v_{i} \partial v_{j}}\left(\left[\delta_{i, j}-\frac{v_{i} v_{j}}{U}\right] \widetilde{F}_{t}^{0}(v)\right),
$$

where $\delta=2 \lambda^{-1} d^{-1}$. Equation (18) is the Master Equation for the SDE (2).

Let $f_{t}^{\varepsilon, N}\left(p_{1}\right)$ be the one particle marginal of $F_{t}^{\varepsilon}(p)$,

$$
f_{t}^{\varepsilon, N}\left(p_{1}\right)=\int F_{t}^{\varepsilon}\left(p_{1}, p_{2}, \ldots, p_{N}\right) d p_{2} \cdots d p_{N}
$$

In [3] it was shown that the limit

$$
f_{t}^{\varepsilon}(p)=\lim _{N \rightarrow \infty} f_{t}^{\varepsilon, N}\left(p_{1}\right)
$$

satisfies the Boltzmann-Vlasov equation

$$
\dot{f}_{t}^{\varepsilon}(p)+\nabla_{p}\left(\left[E-\frac{E j(t)}{U} p\right] f_{t}^{\varepsilon}(p)\right)=\lambda^{-1} \int_{S^{d-1}}\left(f_{t}^{\varepsilon}(|p| \omega)-f_{t}^{\varepsilon}(p)\right) d \sigma(\omega)
$$

where $j(t)$ is fixed by the self-consistent condition

$$
j(t)=\int_{\mathbb{R}^{d}} p f_{t}^{\varepsilon}(p) d p
$$

We can repeat the above scaling analysis by setting $\tilde{f}_{t}^{\varepsilon}(p)=f_{t / \varepsilon^{2}}^{\varepsilon}(p)$ and taking the van Hove limit

$$
\tilde{f}_{t}^{0}(p)=\lim _{\varepsilon \rightarrow 0} f_{t}^{\varepsilon}(p)
$$

A formal perturbative argument very similar to the one used for $F_{t}^{\varepsilon}(p)$ gives that $\tilde{f}^{0}(p)$ depends only on $v=|p|$ and that it satisfies

$$
\dot{\tilde{f}}_{t}^{0}(v)=\delta \frac{d}{d v}\left(v \tilde{f}_{t}^{0}(v)\right)+\frac{\delta}{2} \frac{d^{2}}{d v^{2}} \tilde{f}_{t}^{0}(v)
$$

In Appendix B we show that for large $N$, the speed of an individual particle in (2) is close to an Ornstein-Uhlenbeck process whose Fokker-Planck equation is (19). Thus taking the van Hove scaling $E \rightarrow 0$ and then the large system limit $N \rightarrow \infty$ is, at least formally, equivalent to taking the large system limit before the van Hove scaling. In this sense the van Hove scaling studied in this paper is consistent, at least at a formal level, with the large $N$ limit studied in [3]. 


\section{A Continuity of solution map of differential equations}

Suppose that $x(t), 0 \leq t \leq 1$, is a continuously differentiable path in $\mathbb{R}^{2}$, and that $A: \mathbb{R}^{2} \rightarrow \mathbb{R}^{2 \times 2}$ is a smooth matrix-valued function. Let $y$ be a solution of an integral equation

$$
y(t)=\int_{0}^{t} A(y(s)) d x(s) .
$$

The integral above is understood in the Riemann-Stieltjes sense, and $y$ is uniquely defined.

Let $\left.\Gamma: C^{1}\left([0,1], \mathbb{R}^{2}\right]\right) \rightarrow C^{0}\left([0,1], \mathbb{R}^{2}\right)$ be the solution map for (20). That is, $\Gamma(x)=y$.

It follows from Grönwall's inequality that $\Gamma$ is continuous. So, if a sequence $x^{\varepsilon}$ converges to $x^{0}$ in $C^{1}$ topology as $\varepsilon \rightarrow 0$, then the corresponding sequence $y^{\varepsilon}=\Gamma\left(x^{\varepsilon}\right)$ converges to $y^{0}=\Gamma\left(x^{0}\right)$ in the $C^{0}$ topology.

The domain of $\Gamma$ can be extended to the space $C^{\alpha}$ of $\alpha$-Hölder paths when $\alpha>1 / 2$. (Or alternatively to the space of paths of bounded $p$-variation with $p<2$.) In this case, the integral in (20) is a Young integral [18]. The map $\Gamma$ is still continuous on $C^{\alpha}$, see [17, Theorem 1.28], [12, Section 8.6].

But sample paths of Brownian motions are $\alpha$-Hölder continuous only when $\alpha<1 / 2$, where it is problematic to extend $\Gamma$ in a meaningful way. We illustrate a problem with continuity of possible extensions of $\Gamma$ by the following standard example.

Let

$$
A:\left(\begin{array}{l}
a \\
b
\end{array}\right) \mapsto\left(\begin{array}{ll}
1 & 0 \\
0 & a
\end{array}\right) .
$$

Then $y_{1}=x_{1}$ and $y_{2}(t)=\int_{0}^{t} x_{1}(s) \dot{x}_{2}(s) d s$. Let $x^{\varepsilon}, \varepsilon>0$, be sequence of smooth paths

$$
x^{\varepsilon}(t)=\varepsilon\left(\begin{array}{c}
\cos \left(\varepsilon^{-2} t\right) \\
\sin \left(\varepsilon^{-2} t\right)
\end{array}\right) .
$$

It is easy to see that $x^{\varepsilon}$ converges to $x^{0} \equiv 0$ in $\alpha$-Hölder topology for each $\alpha<1 / 2$ (but not for $\alpha \geq 1 / 2$ ). For small $\varepsilon$,

$$
y^{\varepsilon}(t)=\left(\begin{array}{c}
\varepsilon \cos \left(\varepsilon^{-2} t\right) \\
\int_{0}^{t} \cos ^{2}\left(\varepsilon^{-2} s\right) d s
\end{array}\right) \approx\left(\begin{array}{c}
0 \\
t / 2
\end{array}\right) .
$$

Hence $y^{\varepsilon}$ does not converge to $y^{0}=\Gamma\left(x^{0}\right) \equiv 0$. Thus $\Gamma$ cannot be extended to a continuous map on the space of $\alpha$-Hölder paths, $\alpha<1 / 2$.

Remark A.1. In fact, there is no separable Banach space $\mathcal{B} \subset C^{0}\left([0, \infty), \mathbb{R}^{2}\right)$ such that:

- sample paths of Brownian motions lie in $\mathcal{B}$ almost surely,

- the map $\Gamma$, defined on smooth paths, extends to a continuous map $\Gamma: \mathcal{B} \rightarrow C^{0}\left([0, \infty), \mathbb{R}^{2}\right)$.

See [15, 17] or [12, Proposition 1.1] for details.

\section{B Projections of spherical diffusion}

For each $n \geq 1$, suppose that $W$ is a Brownian motion in $\mathbb{R}^{n}$ with identity covariance matrix. Define a stochastic process $u$ in $\mathbb{R}^{n}$ as a solution of the Stratonovich differential equation

$$
d u=d W-u \frac{u^{*} \circ d W}{n}, \quad u(0)=\xi .
$$


We require that $\xi$ belongs to the sphere $S=\left\{x \in \mathbb{R}^{n}:|x|=n\right\}$. Then $u$ is a diffusion on $S$.

We are interested in statistical behavior of the one dimensional projections of $u$, say the first coordinate $u_{1}$, with large $n$. For the initial condition $\xi$, we fix $\xi_{1}$ independent of $n$ and choose $\xi_{j}$, $j \geq 2$, arbitrarily, deterministic or random independent of $W$.

Theorem B.1. As $n \rightarrow \infty$, the process $u_{1}(t)$ converges weakly (in the uniform topology) to an Ornstein-Uhnelbeck process

$$
d X=d B-\frac{1}{2} X d t, \quad X(0)=\xi_{1},
$$

where $B$ is a standard one-dimensional Brownian motion.

Proof. We write the stochastic differential equation for $u$ in the Itô form [2, pages 137-138]:

$$
d u=d W-u \frac{u^{*} d W}{n}-\frac{n-1}{2 n} u d t
$$

Denote $p=u_{1}$. Then

$$
d p=\left(1-\frac{p^{2}}{n}\right) d W_{1}-\frac{\sqrt{n-p^{2}}}{n} d W^{\prime}-\frac{n-1}{2 n} p d t
$$

where $W^{\prime}$ is a standard one-dimensional Brownian motion, independent from $W_{1}$, which appears as

$$
d W^{\prime}=\frac{\sum_{j \geq 2} u_{j} d W_{j}}{\sqrt{\sum_{j \geq 2} u_{j}^{2}}}=\frac{\sum_{j \geq 2} u_{j} d W_{j}}{\sqrt{n-p^{2}}} .
$$

Let $X$ be the Ornstein-Uhlenbeck process, $d X=d W_{1}-\frac{1}{2} X d t, X(0)=\xi_{1}$. Let $\delta=p-X$. Note that $\delta(0)=0$. We will show that $\delta(t)$ remains small for $t \geq 0$. Write

$$
\begin{aligned}
d \delta & =\frac{-p^{2}}{n} d W_{1}-\frac{\sqrt{n-p^{2}}}{n} d W^{\prime}-\frac{\delta}{2} d t+\frac{p}{2 n} d t \\
& =\frac{1}{\sqrt{n}} d W^{\prime \prime}-\frac{\delta}{2} d t+\frac{p}{2 n} d t,
\end{aligned}
$$

where $W^{\prime \prime}$ is a standard Brownian motion. Another way of writing the above is

$$
\delta(t)=\frac{1}{\sqrt{n}} W^{\prime \prime}(t)+\int_{0}^{t} \frac{p(s)}{2 n} d s-\frac{1}{2} \int_{0}^{t} \delta(s) d s .
$$

By construction, $|p| \leq \sqrt{n}$ at all times. Let

$$
\alpha(t)=|\delta(t)| \quad \text { and } \quad \beta(t)=\frac{\left|W^{\prime \prime}(t)\right|}{\sqrt{n}}+\frac{t}{2 \sqrt{n}} .
$$

Then

$$
0 \leq \alpha(t) \leq \beta(t)+\frac{1}{2} \int_{0}^{t} \alpha(s) d s .
$$

By the Gronwall inequality [14, Lemma 4.5.1],

$$
\alpha(t) \leq \beta(t)+\frac{1}{2} \int_{0}^{t} e^{(t-s) / 2} \beta(s) d s \leq\left(1+\frac{t e^{t}}{2}\right) \hat{\beta}(s),
$$

where $\hat{\beta}(t)=\max _{s \in[0, t]} \beta(s)$. Let also $\hat{W}^{\prime \prime}(t)=\max _{s \in[0, t]}\left|W^{\prime \prime}(s)\right|$. By Burkholder's inequality, $\mathbb{E} \hat{W}^{\prime \prime}(t) \leq C \sqrt{t}$, where $C$ is an absolute constant. Thus $\mathbb{E} \hat{\beta}(t) \leq C(\sqrt{t}+t) / \sqrt{n}$.

We have shown that $p=X+\delta$, where $X$ is the required Ornstein-Uhlenbeck process, and $\mathbb{E}\left(\sup _{s \leq t}|\delta(s)|\right) \leq C_{t} / \sqrt{n}$, where $C_{t}>0$ only depends on $t$. This implies our result. 


\section{Acknowledgements}

This material is based upon work supported by AFOSR under the award number FA9500-16-10037.

A.K. was funded by a European Advanced Grant StochExtHomog (ERC AdG 320977) at the University of Warwick and an Engineering and Physical Sciences Research Council grant $\mathrm{EP} / \mathrm{P} 034489 / 1$ at the University of Exeter.

F.B. gratefully acknowledges financial support from the Simons Foundation award number 359963.

\section{References}

[1] N. Ashcroft and N. Mermin. Solid State Physics. Saunders College, Philadelphia, 1976.

[2] J. F. Blowey, J. P. Coleman, and A. W. Craig, editors. Theory and numerics of differential equations. Universitext. Springer-Verlag, Berlin, 2001. Papers from the 9th EPSRC Numerical Analysis Summer School held at the University of Durham, Durham, July 10-21, 2000.

[3] F. Bonetto, E. A. Carlen, R. Esposito, J. L. Lebowitz, and R. Marra. Propagation of chaos for a thermostated kinetic model. J. Stat. Phys., 154(1-2):265-285, 2014.

[4] F. Bonetto, N. Chernov, A. Korepanov, and J. Lebowitz. Spatial structure of stationary nonequilibrium states in the thermostatted periodic Lorentz gas. Journ, Stat. Phys., 146:1221$1243,2012$.

[5] F. Bonetto, N. Chernov, A. Korepanov, and J. L. Lebowitz. Nonequilibrium stationary state of a current-carrying thermostated system. EPL, 102:15001, 2013.

[6] F. Bonetto, D. Daems, and J. Lebowitz. Properties of stationary nonequilibrium states in the thermostatted periodic Lorentz gas i: The one particle system. Journ, Stat. Phys., 101:35-60, 2000 .

[7] F. Bonetto, D. Daems, J. Lebowitz, and V. Ricci. Properties of stationary nonequilibrium states in the thermostatted periodic Lorentz gas: The multiparticle system. Phys. Rev E, 65:05124, 2002.

[8] J. T. Chang and D. Pollard. Conditioning as disintegration. Statistica Neerlandica, 51(3):287317, 1997.

[9] J. De Simoi and C. Liverani. Statistical properties of mostly contracting fast-slow partially hyperbolic systems. Invent. Math., 206(1):147-227, 2016.

[10] J. De Simoi and C. Liverani. Limit theorems for fast-slow partially hyperbolic systems. Invent. Math., 213(3):811-1016, 2018.

[11] D. Dolgopyat. Averaging and invariant measures. Mosc. Math. J., 5(3):537-576, 742, 2005.

[12] P. K. Friz and M. Hairer. A course on rough paths. Universitext. Springer, Cham, 2014. With an introduction to regularity structures. 
[13] D. Kelly and I. Melbourne. Smooth approximation of stochastic differential equations. Ann. Probab., 44(1):479-520, 012016.

[14] P. E. Kloeden and E. Platen. Numerical solution of stochastic differential equations, volume 23 of Applications of Mathematics (New York). Springer-Verlag, Berlin, 1992.

[15] T. Lyons. On the nonexistence of path integrals. Proc. Roy. Soc. London Ser. A, 432(1885):281-290, 1991.

[16] T. J. Lyons. Differential equations driven by rough signals. Rev. Mat. Iberoamericana, 14(2):215-310, 1998.

[17] T. J. Lyons, M. Caruana, and T. Lévy. Differential equations driven by rough paths, volume 1908 of Lecture Notes in Mathematics. Springer, Berlin, 2007. Lectures from the 34th Summer School on Probability Theory held in Saint-Flour, July 6-24, 2004, With an introduction concerning the Summer School by Jean Picard.

[18] L. C. Young. An inequality of the Hölder type, connected with Stieltjes integration. Acta Math., 67(1):251-282, 1936. 